\title{
Echtzeitfähige Merkmalsextraktion und Situationsinterpretation aus Laserscannerdaten
}

\author{
Kai O. Arras, Sjur J. Vestli, Nadine N. Tschichold-Gürman \\ Institut für Robotik \\ Eidgenössische Technische Hochschule Zürich \\ CH - 8092 Zürich \\ \{arras, vestli, tschichold\}@ifr.mavt.ethz.ch
}

\section{Kurzzusammenfassung}

Ein Verfahren für die Extraktion beliebiger, modellbasierter Merkmale aus Laserscannerdaten wird vorgestellt. Es arbeitet ohne Verwendung von Vorwissen über Ort und Anzahl der zu erkennenden Landmarken und besitzt dabei die Komplexität $O(n)+O\left(l^{2}\right)(n$ : Anzahl Messpunkte, $l$ : Anzahl Umgebungsmerkmale). Seine Implementation zur Erkennung linienhafter Umgebungsstrukturen weist hohe Genauigkeit und Echtzeitfähigkeit auf. Eine Anwendung in der Positions- und Orientierungsbestimmung mobiler Roboter mittels eines Erweiterten Kalman Filters liegt vor.

Die darauf aufbauende Interpretationsmethode für strukturierte Indoor-Situationen liefert eine geometrische und symbolische Situationsbeschreibung. Diese ermöglicht eine im Sinne der Topologie richtige Situationsinterpretation, mit welcher konkave und konvexe Ecken, Öffnungen und potentielle Öffnungen erkannt werden können. Sie eignet sich zur Situationsklassifikation, zur situationsspezifischen Verhaltensauswahl oder zur Anweisung eines Explorationsalgorithmus, womit sowohl der metrische als auch der rein topologische Ansatz eines anschliessenden Map Buildings offensteht.

\section{Einleitung}

Die Frage nach der Umwelterkennung und Sensordateninterpretation ist wesentlicher Kern jeder Anwendung autonomer mobiler Roboter. Die Einsatzgebiete solcher Systeme weisen in der Regel einen gewissen Grad der Strukturiertheit auf, der sowohl von Gebäudemerkmalen als auch von Einrichtungsgegenständen herrührt. Für die Lösung des Problems von Navigation und Map Building ist die Extraktion geometrischer Primitive ein möglicher Weg, der hier durch die Detektion beliebiger, modellbasierter Umgebungsstrukturen beschritten werden soll.

Die Erkennung topologierelevanter Situationen und die Gewinnung metrischer Information sind zentrale Fragestellungen beim Kartographieren unbekannter Umgebungen. Verschiedene Methoden wurden für die Modellierung der Umwelt vorgeschlagen, welche sich in zwei sich gegenseitig nicht ausschliessende Klassen einteilen lassen. Es sind dies der metrische und der rein topologische Ansatz. Die Anwendung der hier vorgestellten Methode impliziert keine Festlegung auf eine dieser Ansätze. Das hier vorgestellte Verfahren der Situationsbeschreibung besteht aus der Ansammlung geometrischen Wissens über den betrachteten Umgebungsausschnitt und aus einer symbolischen String-Beschreibung, die auf hohem Abstraktionsniveau Aussagen über die Topologie der Situation zulässt. 


\section{Das Extraktionsverfahren}

Im folgenden Abschnitt wird anfänglich die Extraktion von Geraden als eines der wichtigsten Landmarken beschrieben, das ausserdem von zentraler Bedeutung für die nachfolgende Situationsbeschreibung ist. Aus Gründen der Anschaulichkeit wird erst am Schluss die Verallgemeinerung für beliebige Umgebungsmerkmale vorgenommen.

Das Problem wird nach der Vorverarbeitung als erstes in ein Clusteringproblem umgewandelt. Der zentrale Teil ist ein wohlbekannter Clusteranalysealgorithmus, ein agglomeratives, hierarchisches Gruppierungsverfahren. Dieser Algorithmus mit der Komplexität $O\left(l^{2}\right)$ kann in einer Echtzeitanwendung nur durch erhebliche Reduktion der Eingangsinformation sinnvoll angewendet werden, was durch die vorhergehende Gewinnung der beitragenden Segmente erreicht wird. Schliesslich wird die Frage nach dem idealen Clustermittelpunkt optimal hinsichtlich einer präzisen Bestimmung der Geradenparameter beantwortet.

\subsection{Vorverarbeitung}

Die Vorverarbeitung der Rohdaten umfasst drei Schritte, welche gemeinsam das Ziel verfolgen, die Anzahl Messpunkte bei kleinstmöglichem Informationsverlust zu verringern: Der erste Durchgang verwirft Punkte, die ausserhalb eines festgelegten Abstandsintervalls liegen (zu weit weg oder zu nahe), der zweite entfernt alleinstehende Messungen und der dritte dünnt die Daten so aus, dass benachbarte Punkte in einer Mindestdistanz zueinander entfernt liegen [VESTLI95]. Eine vorverarbeitete Aufnahme eines Korridors mit Verzweigung ist in Figur 2-1 abgebildet.

Mit den vier, hier zu definierenden Parametern wird auf die Spezifikation des verwendeten Laserscanners eingegangen. Die Winkelauflösung, diejenige in radialer Richtung sowie der Charakter des Messrauschens sind die massgebenden Grössen des Sensors.

\subsection{Gewinnung der Clusters im Parameterraum}

Durch jeweils drei unmittelbar benachbarte Punkte $\left\{P_{i-1}, P_{i}, P_{i+1}\right\}$ wird eine Gerade im Sinne kleinster Fehlerquadrate eingelegt. Diese wird mittels den Parametern $\alpha$ und $r$ in der Hesseschen Normalform der Geradengleichung beschrieben

$$
x \cos (\alpha)+y \sin (\alpha)-r=0,
$$

wobei $r$ die Länge des Lots von der Geraden zum Urprung angibt und $\alpha$ dessen Winkel mit der $\mathrm{x}$-Achse des Sensorkoordinatensystems. Eine effiziente Berechnungsvorschrift für die Modellparameter $\alpha$ und $r$ wird in [KANA89] vorgestellt.

Wenn nun mehrere Messpunkte in etwa auf einer Geraden liegen, so nehmen ihre assoziierten $\alpha / r$-Parameter auch ähnliche Werte an, was sich in der Ausbildung homogener Bereiche in der Darstellung von $\alpha$ und $r$ gegen den Messindex äussert (dünne Kurven in Figur 2-2). Wechselt man die Darstellungsart und trägt $\alpha$ gegen $r$ ab, so erscheinen diese Bereiche jetzt als Clusters im Parameterraum, die jeweils zu einer der gesuchten linienhaften Strukturen korrespondieren (Punkte in Figur 2-3).

Damit konnte das Extraktionsproblem in ein Clusteringproblem umformuliert werden, wobei evident ist, dass weder Ort noch Anzahl der zu erwartenden Clusters bekannt sind. Einfache Hochrechnungen zeigen, dass der Einsatz herkömmlicher Methoden der Clusteranalyse an diesem Ort zu unpraktikabel hohen Rechenzeiten führen würde. Es muss daher eine Eigenschaf gefunden werden, mittels derer sich dieses Problem von einem allgemeinen Clusteringproblem unterscheidet. 


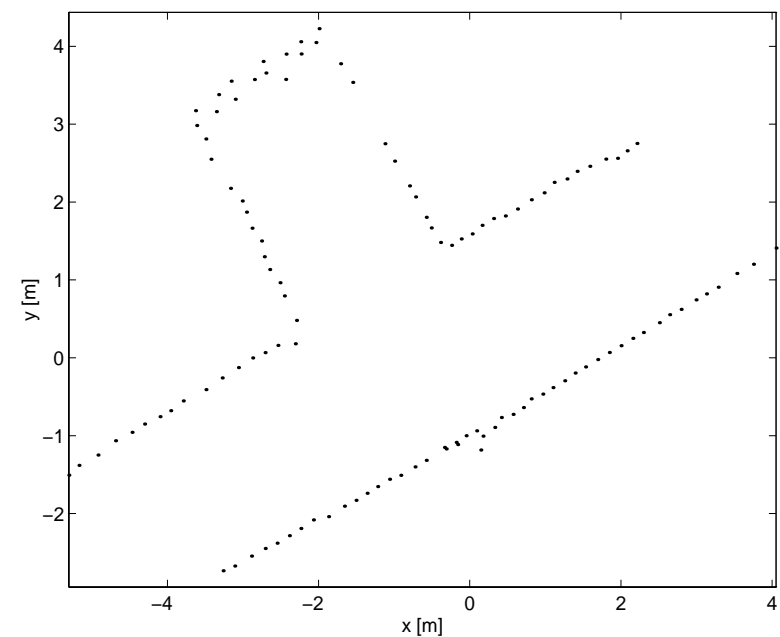

Figur 2-1: Vorverarbeiteter Scan, aufgenommen in einem Korridor mit Verzweigung.

Im ersten Schritt wird durch drei unmittelbar benachbarte Messpunkte des Scans eine Gerade im Sinne kleinster Fehlerquadrate eingelegt und mittels den Parametern $\alpha$ und $r$ in der Hesseschen Normalform der Geradengleichung beschrieben.

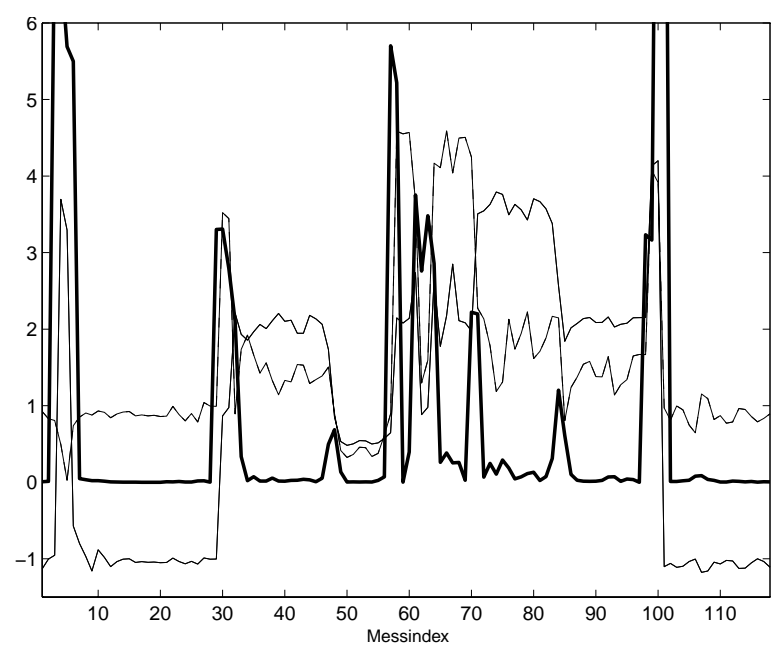

Figur 2-2: Assoziierte $(\alpha, r)$-Wertepaare aufgetragen gegen den Messindex (dünne Kurven). Dort, wo Punkte auf einer Wand liegen, bilden sich sowohl in $\alpha$ als auch in $r$ homogene Bereiche heraus.

Dick ausgezogen das Kompaktheitsmass $e_{i}$. In den Gebieten ähnlicher $\alpha / r$-Werte ist $e_{i}$ erwartungsgemäss nahe bei Null.

\subsection{Bestimmung der beitragenden Segmente}

Diese Eigenschaft gibt es und sie manifestiert sich als Nachbarschaftsrelation der Messpunkte: Der Aquisition der Messpunkte unterliegt eine Systematik durch die Tatsache, dass der Laserscanner die Umgebung in einer wohldefinierten Rotationsrichtung abtastet und nicht Messungen in beliebigen Winkeln und zufälliger Reihenfolge macht. Dieser Systematik ist es zu verdanken, dass sich die in Figur 2-2 beobachteten homogenen Bereiche herausbilden, die damit im Gegensatz zu einem gänzlich unstrukturierten Auftreten der $(\alpha, r)$-Werte stehen.

Die Nachbarschaftsrelation lässt folglich die Aussage zu, dass Messpunkte auf Geraden nacheinander auftreten und somit nebeneinander im Verbund der Messungen abgelegt werden. Oben wurde zudem die Feststellung gemacht, dass auf Geraden liegende Messpunkte sich durch die Clusterbildung im Parameterraum auszeichnen. Diese zwei Aussagen ermöglichen nun den einfachen Schluss, dass die gesuchten, sich in Clustern befindlichen Punkte, nebeneinander anzutreffen sind.

Das eröffnet die im folgenden ausgenützte Möglichkeit, die Clusters aufzuspüren. Wir richten dafür unsere Aufmerksamkeit wiederum auf den $\alpha / r$-Raum sowie auf ein zu definierendes Distanzmass, das die Nähe von darin befindlichen Punkten quantifiziert. Ein bekanntes Distanzmass für diesen Zweck ist die Abweichungsquadratsumme vom Mittelwertsvektor (Gl. 2-2), welches als ein Kompaktheitsmass eines Clusters $C_{j}$ angesehen werden kann [SPÄTH83].

$$
e\left(C_{j}\right)=\sum_{i \in C_{j}}\left\|x_{i}-\bar{x}_{j}\right\|^{2}, \quad x_{i} \in \mathfrak{R}^{s}
$$

Dieses tritt im Kontext des Varianzkriteriums auf, welches in der Clusteranalyse als mögliche Gütefunktion einer gegebenen Partition Verwendung findet, die es durch nachgeschaltete iterative Verfahren zu optimieren gilt. Hier interessiert uns nur die Kompaktheit dreier benachbarter Punkte $\left\{x_{i-1}, x_{i}, x_{i+1}\right\}$, womit Gleichung 2-2 umgeschrieben werden kann zu

$$
e_{i}=\sum_{j=i-1}^{i+1}\left\|x_{j}-\bar{x}_{i}\right\|^{2}, \quad \bar{x}_{i}=\frac{1}{3} \sum_{j=i-1}^{i+1} x_{i} .
$$


Hier gilt natürlich $x_{i}=(\alpha, r)_{i}$ und damit $s=2$.

Dieser Wert ist offensichtlich dann nahe bei Null, wenn die drei Punkte dicht beieinander liegen. Trägt man ihn gegen den Messindex ab, so erscheint eine Kurve, die durch ihr Rausch- und Diskriminanzverhalten die homogenen Bereiche des $\alpha$ - und $r$-Graphen gut abtrennt (dick ausgezogene Kurve in Figur 2-2).

Ein beitragendes Segment ist nun definiert als Menge von Punkten, deren Indizes einen zusammenhängenden Bereich unterhalb des Schwellwertes $\varepsilon$ bilden. Markiert man die so erhaltenen Punkte im ursprünglichen Scan, so wird ersichtlich, dass Punkte gefunden wurden, welche in Regionen liegen, die den Verlauf des gemessenen Linienstücks gut angeben (hervorgehobene Punkte in Figur 2-4).

Im allgemeinen kann jedoch dasselbe Umgebungsmerkmal durch mehrere beitragende Segmente repräsentiert sein. Die endgültige Lagebestimmung der Gerade sollte aber für bestmögliche Genauigkeit die Punkte aller Segmente in Betracht ziehen. Das Clusteringproblem ist demzufolge noch nicht gelöst und verlangt, diejenigen Segmente zu assoziieren, welche zu derselben linienhaften Umgebungsstruktur gehören.

\subsection{Der Clustering-Algorithmus und die endgültige Lagebestimmung}

Dafür wird für jedes beitragende Segment eine Annäherung der endgültigen Geradenparameter bestimmt. Diese Approximation erhält man durch ein erneutes Einpassen einer Gerade im Sinne kleinster Fehlerquadrate - jetzt jedoch durch jeweils alle Punkte des beitragenden Segments. So erhält man pro Segment einen Punkt im Parameterraum, die im Kollektiv an jenen Stellen clu-

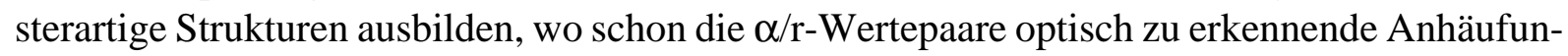
gen zeigten (Kreise in Figur 2-3). Was erreicht wurde, ist eine erhebliche Reduktion der Eingangsinformation, was erst jetzt die Anwendung bekannter Verfahren zur Clusteranalyse eröffnet, die den hohen Anforderungen des Problems hinsichtlich Echtzeitfähigkeit bei begrenzter Rechenleistung gerecht wird.

Die angewandte Gruppierungsmethode für die Assoziation der zum selben Linienstück gehörenden Segmente ist ein agglomeratives, hierarchisches Clusteringverfahren [HARTIG75], [SPÄTH83], was sich leicht und effizient implementieren lässt. Die Abstandsfunktion ist euklidisch, als Aktualisierungsmethode der Distanzmatrix wurde das konservative und monotone Average-Linkage gewählt.

Nach der Anwendung dieses Schritts, ist die Frage, $o b$ ein Punkt zur Lagebestimmung der Gerade beiträgt, geklärt, d.h. die Korrespondenz von Messpunkt zu Merkmal ist hergestellt. Schliesslich verbleibt das Problem, wie ein Punkt zur Lagebestimmung beiträgt, was der Bestimmung des Clusterzentrums entspricht, das optimal hinsichtlich der ursprünglichen Fragestellung berechnet werden soll. Dazu wird wiederum eine Gerade im Sinne kleinster Fehlerquadrate eingepasst - diesmal jedoch durch alle Punkte, die einem beobachteten Linienstück zugeordnet sind.

In Figur 2-4 sind die endgültig extrahierten Geraden eingezeichnet. Die kurze Wand im oberen Teil der Figur wurde aufgrund dem starken Rauschen ihrer Messpunkte nicht extrahiert. Dies kann auch im Parameterraum eingesehen werden (Figur 2-3), wo dem im oberen Teil alleine liegende Cluster kein Zentrum (dicker Pfeil) zugeordnet ist.

\subsection{Verallgemeinerung}

Es ist offensichtlich erstrebenswert, verschiedene Merkmale extrahieren zu können, um einen höheren Grad der Allgemeinheit zu erlangen. Es lässt sich einsehen, dass das vorliegende Ver- 


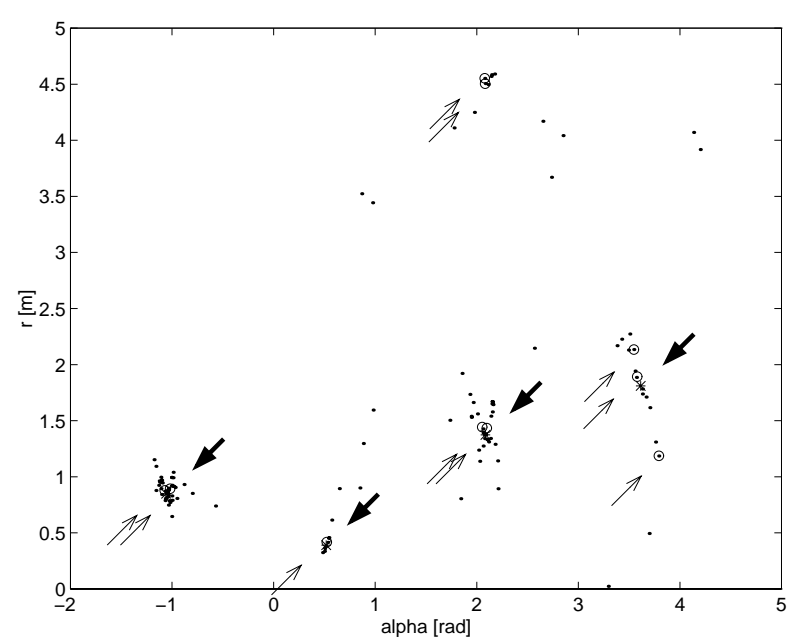

Figur 2-3: Clusters im Parameteraum, die zu den gesuchten Geraden korrespondieren.

Punkte: $\alpha / \mathrm{r}$-Clusters

Kreise: Segmentclusters (dünne Pfeile)

Sterne: Endgültige Clusterzentren (dicke Pfeile)

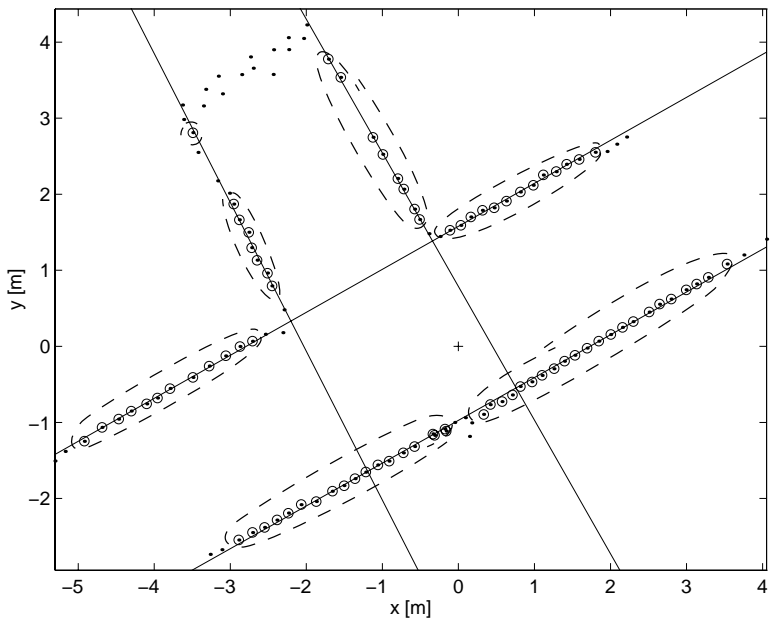

Figur 2-4: Beitragende Segmente, markiert in der Darstellung des ursprünglichen Plots. Die hervorgehobenen Punkte sind offenbar solche, die die Richtungen der Wände gut angeben. Ferner eingezeichnet sind die endgültigen Lagen der vier extrahierten Geraden.

fahren Allgemeingültigkeit besitzt und sich für die Extraktion beliebiger, modellbasierter geometrischer Primitive eignet. Verfolgen wir nun die eben kennengelernten Schritte für allgemeine Merkmale:

- Schritt 1: Lege das Merkmalsmodell im Sinne kleinster Fehlerquadrate in $m$ benachbarte Messpunkte ein. Es bilden sich homogene Bereiche in den Kurven der Modellparameter aus, die mit den aufgenommenen Merkmalen korrespondieren. Trägt man die Parameter gegeneinander $a b$, entstehen Clusters im Parameterraum.

- Schritt 2: Wende ein Distanzmass auf benachbarte Punkte im Parameterraum an, um die Clusters aufzuspüren (Ausnützen der Nachbarschaftsrelation). Trägt man diese Distanz gegen den Messindex ab, zeichnen sich die Orte der Clusters durch zusammenhängende Gebiete sehr geringer Distanz aus. Die beitragenden Segmente erhält man schliesslich durch Anwenden eines Schwellwertes $\varepsilon$, der diese Gebiete isoliert.

- Schritt 3: Berechne eine Approximation der endgültigen Modellparameter für jedes beitragende Segment. Benutze diese dadurch entstehenden Punkte im Parameterraum als Eingang des agglomerativen, hierarchischen Gruppierungsverfahrens, welche die definitive Korrespondenz von Messpunkt zu Merkmal herstellt.

- Schritt 4: Bestimme die Clusterzentren durch erneutes Einpassen des Modells im Sinne kleinster Fehlerquadrate in die jetzt bekannten Menge von Messpunkten. Die endgültigen Parameter stehen somit fest.

Die Grösse $m$ hängt von der Winkelauflösung des verwendeten Sensors ab. Bei hoher Winkelauflösung kann man sich den Detailverlust der stärker werdenden Glättung bei grossem $m$ leisten. Bei niedriger Auflösung wird man von Vorteil ein detailerhaltendes, aber zugleich noch minimal glättendes $m$ wählen.

Der Schwellwert $\varepsilon$ steht in reiner Abhängigkeit des Sensorrauschens in radialer Richtung. Besitzt der eingesetzte Sensor geringe Genauigkeit, wird man $\varepsilon$ vergrössern. Das führt jedoch zu einer Steigerung des Risikos von Fehlklassifikationen, da Umgebungsstrukturen mit ähnlicher Form fälschlicherweise als die gesuchten Merkmale interpretiert werden können. Bei Sensoren hoher Güte, kann $\varepsilon$ vermindert werden, so dass nur Umgebungsstrukturen mit sehr grosser 
Formtreue extrahiert werden. Hier muss ein Kompromiss zwischen geforderter Modelltreue der extrahierten Merkmale und dem Risiko von Fehlklassifikationen gefunden werden.

\subsection{Ergebnisse}

Es liegen Ergebnisse für Genauigkeit und Geschwindigkeit der Geradenextraktion vor. Diese beziehen sich auf die verwendete Hardware eines Motorola 68020 bei $20 \mathrm{MHz}$. Beim Laserscanner handelt es sich um einen Leuze Rotoscan RS 3 mit $2^{\circ}$ Winkelauflösung und $4 \mathrm{~mm}$ radialer Auflösung. Die Standardabweichung in radialer Richtung beträgt ca. $7 \mathrm{~mm}$. Der Roboter stand in einer Ecke mit zwei gut sichtbaren Wänden, wo 300 Messungen vorgenommen wurden. Es konnten folgende Werte für das Rauschen der Lageparameter der extrahierten Geraden identifiziert werden:

- Standardabweichung in $\alpha: \sigma_{\alpha}=0.2^{\circ}$, Spannweite in $\alpha: s_{\alpha}=1.5^{\circ}$

- Standardabweichung in $r: \sigma_{r}=5 \mathrm{~mm}$, Spannweite in $r: s_{r}=2.5 \mathrm{~cm}$

- Rechenzeit bei 90 prozessierten Messpunkten (inkl. Vorverarbeitung): < $240 \mathrm{~ms}$.

- Rechenzeit für die Erhaltung der Situationsbeschreibung (siehe unten): $<10 \mathrm{~ms}$

\section{Geometrische und symbolische Situationsbeschreibung}

Eine für das Map Building wesentliche Frage ist die Erkennung von Öffnungen oder allgemeiner, die der topologierelevanten Situationen. Es ist evident, dass ein Fehler in der Topologie eine schwerwiegende Unzulänglichkeit der Karte darstellt, was die Notwendigkeit einer im Sinne der Topologie korrekten Interpretation von Situationen verdeutlicht. Eine Situationsbeschreibung wird auch dann unumgänglich, wenn man auf den Einbezug metrischer Information in die Karte verzichten will und statt dessen ein rein topologiegeführtes Map Building- und Navigationskonzept verfolgt wie es z.B. in [KUIPERS88] vorgeschlagen wurde.

Hier sind wir in der Lage, eine geometrische und symbolische Beschreibungsart für strukturierte Indoor-Umgebungen zu entwickeln. Die gesamte Situationsbeschreibung basiert auf dem Schneiden benachbarter, linienhafter Umgebungsstrukturen und der Klassifikation des dabei entstehenden Schnittpunktes. Dazu wird auf die Ebene der beitragenden Segmente zurückgegriffen, da sie die Information über die Nachbarschaft aller extrahierten Merkmale enthält.

Ein für die erfolgreiche Unterscheidung erforderliches Klassifikationsmerkmal ist die Lage des gemeinsamen Schnittpunktes $C_{i, i+1}$, der entsteht, wenn die Geraden der benachbarten Segmente $S_{i}$ und $S_{i+1}$ geschnitten werden. In einer vorübergehenden Vereinfachung lässt sich sagen: wenn zwei Geraden parallel zueinander stehen und ihr Schnittpunkt im Unendlichen liegt, ergibt sich eine Öffnung, wenn er im Endlichen liegt eine konkave oder konvexe Ecke. Die hier zweckmässige Definition von "unendlich" und "endlich" lässt sich an Figur 3-1 verdeutlichen: Ein Schnittpunkt ist im "Unendlichen", wenn er ausserhalb des Kreises $K_{R}$ liegt, andernfalls im "Endlichen". Der Kreisradius $r_{\max }$ ist ein in der Vorverarbeitung schon verwendeter Parameter, der den Rezeptionsradius des Roboters festlegt, indem alle Messpunkte mit grösserem Radialwert verworfen werden. Der Roboter "sieht" demnach nichts, was weiter weg ist als $r_{\text {max }}$.

In Figur 3-1 sind überdies alle möglichen Fälle abgebildet, die beim Schneiden benachbarter Segmentgeraden auftreten können. Abgebildet ist die schematische Darstellung einer Situation mit fünf extrahierten Segmenten $S_{1}, S_{2}, \ldots, S_{5} ; R$ bezeichnet die Roboterposition. Die Umgebungsgeraden sind dünn ausgezogen, die beitragenden Segmente durch dicke Linien hervorgehoben. Die drei ersten Schnittpunkte - eine konvexe Ecke $\left(C_{1,2}\right)$, eine konkave Ecke $\left(C_{2,3}\right)$ und eine Öffnung $\left(C_{3,4}\right)$ - sind alle noch mit einfachen Mitteln unterscheidbar. Die zwei letzten Fälle 


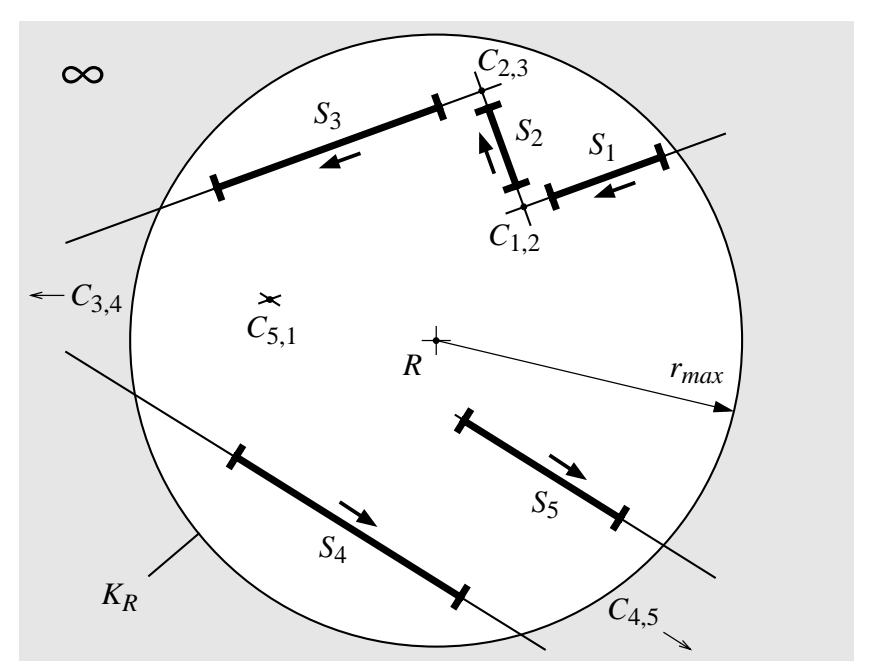

Figur 3-1: Alle möglichen Fälle beim Schneiden benachbarter Segmentgeraden. Mit einfachen Mitteln unterscheidbar sind die drei ersten Schnittpunkte: $C_{1,2}$ konvexe Ecke, $C_{2,3}$ konkave Ecke und $C_{3,4}$ Öffnung. Die zwei letzten Fälle benötigen die Direktionalität eines Segmentes $\lambda$ als Klassifikationsmerkmal. Damit lassen sich $C_{4,5}$ als verdeckte Ekken und $C_{5,1}$ als Öffnung (obwohl $C_{5,1}$ im Endlichen liegt) identifizieren.

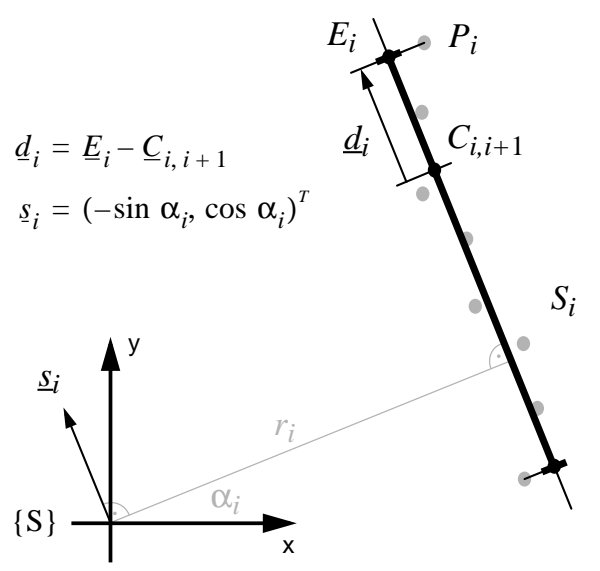

Figur 3-2: Bestimmung der Direktionalität eines Segmentes. Der Schnittpunkt $C_{i, i+1}$, der durch das Schneiden benachbarter Segmentgeraden entsteht, kann irgendwo auf der Segmentgerade liegen. Hier eingezeichnet ist der Fall, bei dem er sich innerhalb von $S_{i}$ befindet. Die Direktionalität $\lambda$ kann schliesslich als einfache Funktion von $\underline{s}_{i}$ und $\underline{d}_{i}$ angegeben werden (Gl. 3-1).

jedoch, bei denen es sich um zwei Öffnungen handelt - halb verdeckt bei $C_{4,5}$ und obwohl der Schnittpunkt im Endlichen liegt auch bei $C_{5,1}$, können nicht ohne weiteres als solche erkannt werden. Sie erfordern das im folgenden beschriebene Klassifikationsmerkmal der Direktionalität eines Segmentes.

\subsection{Die Direktionalität eines Segmentes}

Die Direktionalität eines Segmentes $\lambda$ ist definiert als die Scanrichtung bezüglich des gemeinsamen Schnittpunktes $C_{i, i+1}$. Es wird unterschieden zwischen weg vom Schnittpunkt (markiert durch einen Pfeil, der nach oben, vom Boden weg zeigt: $\uparrow$ ) und hin zum Schnittpunkt (angedeutet mit einem Pfeil, der nach unten, zum Boden hin zeigt: $\downarrow$ ). Die Scanrichtung eines Segmentes wird von derjenigen des Laserscanners bestimmt, die wohlbekannt entweder im Uhrzeigersinn oder im Gegenuhrzeigersinn definiert ist. Dadurch wird allen erkannten Umgebungs-merkmalen eine Richtung aufgeprägt und mittels der Erscheinungsreihenfolge können Aussagen über ihre Nachbarschaft gemacht werden.

Für die konkrete Bestimmung von $\lambda$ betrachte man Figur 3-2. Gegeben sind die Lageparameter der Segmentgerade $\left(\alpha_{i}, r_{i}\right)$, die Koordinaten des Schnittpunktes $C_{i, i+1}$ mit der Geraden des folgenden, benachbarten Segmentes $S_{i+1}$ und die Lage des letzten, dem Segment $S_{i}$ zugehörigen Messpunkt $P_{i}$, welcher an demjenigen Ende von $S_{i}$ liegt, das sich auf der Seite von $S_{i+1}$ befindet. Den Segmentendpunkt $E_{i}$ erhält man dann durch Projektion von $P_{i}$ auf die Segmentgerade. Damit berechnet sich $\underline{d}_{i}$ als Differenz der Vektoren $\underline{E}_{i}$ und $\underline{C}_{i, i+1}$. Die Scanrichtung $\underline{s}_{i}$ kann für einen im Gegenuhrzeigersinn messenden Laserscanner durch Drehen des Lotes um $+\pi / 2$ erhalten werden. Die Verwendung von $P_{i}$ stellt den einzigen, bei der gesamten Situationsbeschreibung notwendigen Rohdatenzugriff dar.

Die Direktionalität $\lambda_{i}$ von Segment $S_{i}$ berechnet sich schliesslich aus der folgenden, einfachen Vorschrift:

$$
\lambda_{i}=\frac{\underline{s}_{i}^{T} \underline{d}_{i}}{\left|\underline{d}_{i}\right|}=\left\{\begin{array}{r}
1, \operatorname{weg}(\uparrow) \\
-1, \operatorname{hin}(\downarrow)
\end{array}\right.
$$


(a) Korridorverengung, kurze

Wand nicht sichtbar: ' $\mathrm{OHO}$ '

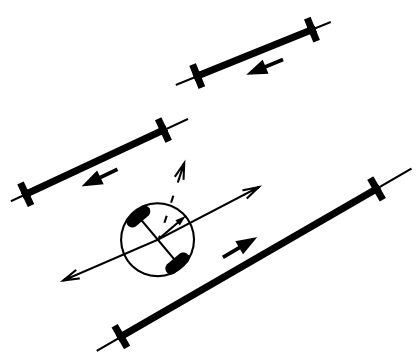

(c) Sackgasse mit zwei Verzweigungen, eine verdeckt:

'OEHCC'
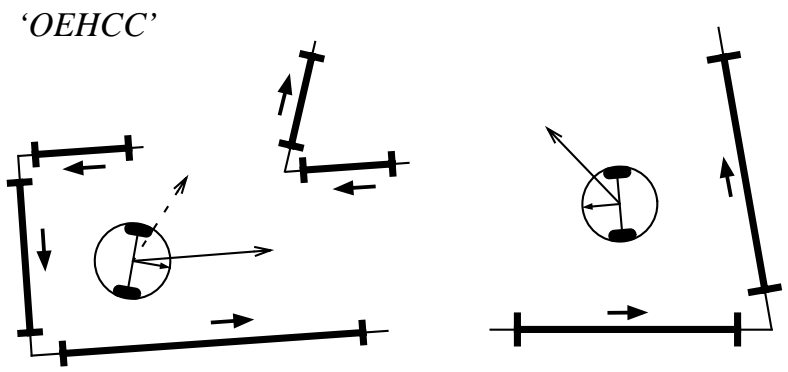

Figur 3-3: Schematische Darstellung einiger Situationen und ihre symbolische String-Beschreibung: Die Wände sind dünn ausgezogen, die beitragenden Segmente durch dicke Linien hervorgehoben. den Ast: ' $H C O$ '

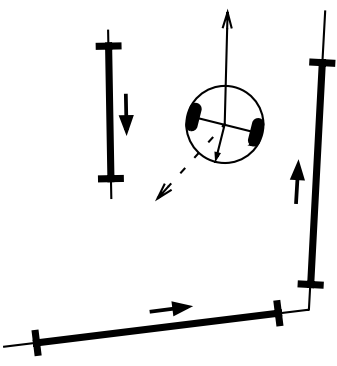

(d) In einer Ecke: ' $O C$ ' (b) L-Biegung, im liegen-

Figur 3-4: Klassifikationsschema für die Unterscheidung der vier Schnittpunktstypen, welches für jedes benachbarte Segmentpaar $S_{i}$ und $S_{i+1}$ durchlaufen wird.

Die in Figur 3-1 eingezeichneten Pfeile geben die Scanrichtungen der Segmente für einen im Gegenuhrzeigersinn messenden Laserscanner an. Mit ihrer Hilfe lassen sich jetzt die Direktionalitäten der Segmente in der dargestellten Situation einfach ermitteln. Begonnen wird mit den Segmenten $S_{1}$ und $S_{2}$ : Der Pfeil von Segment $S_{1}$ zeigt zum Schnittpunkt $C_{1,2}$ hin, also ist $\lambda_{1}$ als hin $(\downarrow)$ definiert, $\lambda_{2}$ als weg $(\uparrow)$. Analog die Fälle von $C_{2,3}$ und $C_{3,4}$. Beim Schneiden der Segmente $S_{4}$ und $S_{5}$ zeigt sich das erste Mal eine Anomalie in den Direktionalitäten. Sie ergeben sich beide als $\operatorname{hin}(\downarrow)$ zum Schnittpunkt $C_{4,5}$. Auch $C_{5,1}$ zeichnet sich wiederum durch abweichende $\lambda$-Werte aus, sie lassen sich als weg $(\uparrow)$ bei $\lambda_{5}$ und hin $(\downarrow)$ bei $\lambda_{1}$ identifizieren. Diese Wertekombinationen der Direktionalitäten sind für beide betrachteten Fälle charakteristisch. In [ARRAS96] wird hierzu eine detailliertere Diskussion geführt, wo durch die Definition einer geeigneten Notation die leichte Unterscheidung aller Fälle ermöglicht wird.

Die Direktionalität eines Segmentes ist somit ein Klassifikationsmerkmal, welches die Formulierung hinreichender Bedingungen für Fälle verdeckter Öffnungen und Öffnungen mit im Endlichen liegenden Schnittpunkten erlaubt. Die Bezeichnung "verdeckte Öffnung" ist im allgemeinen jedoch eine falsche Interpretation. Es sind Ecken, die verdeckt werden und zwar diejenigen, welche die fraglichen zwei Segmente verbinden. Betrachten wir dazu die Korridorverengung in Figur 3-3 (a). Es kann aus der Sicht des Roboters nicht entschieden werden, ob eine kurze Wand oder eine Öffnung verdeckt wird. In beiden Möglichkeiten werden jedoch Ecken verdeckt, eine konkave und eine konvexe im Falle der kurzen Wand und beliebig viele im Falle der Öffnung.

Die symbolische Beschreibung erhält man nun dadurch, dass jeder Ecke ihr entsprechendes Zeichen aus einem Alphabet von vier Buchstaben zugeordnet wird. Das Alphabet besteht aus den Zeichen ' $E$ ' für konvexe Ecke (edge), ' $C$ ' für konkave Ecke (corner), ' $O$ ' für Öffnung (opening) und ' $H$ ' für verdeckte Ecken (hidden corners). Somit ergibt sich ein String variierender 
Länge, der die Situation auf einem sehr hohen Abstraktionsniveau beschreibt. Das endgültige Klassifikationsschema, welches für jedes benachbarte Segmentpaar $S_{i}$ und $S_{i+1}$ durchlaufen wird, ist in Figur 3-4 dargestellt. Die letzte Bedingung für die Unterscheidung konkaver und konvexer Ecken bedient sich der Differenz $\Delta \alpha$, welche durch $\Delta \alpha=\alpha_{i+1}-\alpha_{i}$ definiert ist. In Figur 3-3 sind weitere Situation mitsamt ihrer symbolischen String-Beschreibung dargestellt.

Die geometrische Beschreibung besteht in den Angaben jeweils adäquater geometrischer Grössen: Aus dem vorhergehenden Schritt sind die Lage aller linienhaften Umgebungsstrukturen mitsamt ihren Endpunkten bekannt. Im Falle von konkaven und konvexen Ecken werden Eckposition und Winkel berechnet, bei Öffnungen und verdeckten Ecken bestimmt man die Richtungen, in denen sie liegen.

Damit liegt schliesslich eine Fülle von Information über die momentane Situation des Roboters vor, welche vielfältige Anwendungen eröffnet. Die Anweisung eines Explorationsalgorithmus für unbekannte und komplexe aber bedingt strukturierte Umgebungen ist die hervorstechende Einsatzmöglichkeit der symbolischen String-Beschreibung. Die Implementation einer situationsspezifische Verhaltensauswahl, welche in Abhängigkeit des vorliegenden Umgebungsausschnitts ein angemessenes Fahrverhalten auswählt oder anwendungsspezifische Sonderverhalten aktiviert, ist ebenfalls denkbar.

\section{Anwendung in der Positions- und Orientierungsbestimmung mobiler Roboter}

Im hier angewandten, echtzeitfähigen Lokalisierungsverfahren kommt ein Erweitertes Kal-man Filter zum Einsatz. Diese Möglichkeit wird schon vielerorts verfolgt, z.B. in [LEON92]. Dort werden aus Ultraschalldaten mehrere geometrischer Primitive - Linien, Ecken und Zylinder extrahiert, allerdings ohne Echtzeitfähigkeit zu erlangen. Dennoch bestehen aufgrund gleicher Merkmalsmodelle für die Geraden Ähnlichkeiten, insbesondere in der Beobachtungsvorhersage (measurement prediction). Auch in [VESTLI95] werden obige Landmarken gefunden, jedoch unter Einsatz von Vorwissen über ihren Ort und ihre Anzahl. Mit der Implementation der Geradenextraktion wird Echtzeitfähigkeit erreicht.

Es konnte durch eine gute Harmonisierung mit dem hier eingesetzten, unterlagerten Positionsregler für nichtholonome mobile Roboter [KAISER95] ein ansprechendes Fahrverhalten realisiert werden. Wiederum liegen Resultate für Genauigkeit und Geschwindigkeit vor: Bei stehendem Roboter mit zwei gut sichbaren Wänden rauscht die Positions- und Orientierungsschätzung in $x$ und $y$ mit $6 \mathrm{~mm}$ Standardabweichung, in $\theta$ mit guten $0.05^{\circ}$. Die Spannweiten betragen $2.5 \mathrm{~cm}$ in $x$ und $y, 1.5^{\circ}$ in $\theta$. Bei einer kleinen Tour durch den Laborraum, wo 160 Zyklen durchlaufen wurden, lag die durchschnittliche Zykluszeit bei $445 \mathrm{~ms}$. Wiederum mit der schon erwähnten Hardware eines M68020 bei 20 MHz, auf der jedoch mittels eines Echtzeitbetriebssystems alle laufenden Prozesse quasi-parallel ausgeführt wurden.

\section{Zusammenfassung und Ausblick}

Es wurde ein neues Verfahren zur Extraktion beliebiger modellbasierter Merkmale aus Laserscannerdaten vorgestellt, das ohne Vorwissen über die Umwelt auskommt und bei der Geradenextraktion hohe Genauigkeit und Echtzeitfähigkeit aufzeigt. Das Verfahren besitzt die Komplexität $O(n)+O\left(l^{2}\right)$ ( $n$ : Anzahl Messpunkte, $l$ : Anzahl Umgebungsmerkmale). Die so gewonnenen Lageparameter der linienhaften Umgebungsstrukturen wurden in einem Kalman Filter-ba- 
sierten Verfahren zur Positions- und Orientierungsbestimmung erfolgreich eingesetzt.

Ausserdem wurde eine geometrische und symbolische Situationsbeschreibung vorgestellt, die mittels der eingeführten Direktionalität eines Segmentes elegant hergeleitet werden konnte. Diese ermöglicht eine im Sinne der Topologie richtige Situationsinterpretation, mit welcher konkave und konvexe Ecken, Öffnungen, potentielle Öffnungen und Öffnungen mit im Endlichen liegenden Schnittpunkten erkannt werden können.

Bis jetzt wurde die analytische Diskussion der Fehlerfortpflanzung im Extraktionsalgorithmus ausgelassen. Den Abstandswerten des Sensors wurde volles Vertrauen zugebilligt, was zu fehlerfreien und unkorrelierten Modellparametern führte. In Wahrheit besitzen die Abstandswerte Unsicherheiten, die sich fortpflanzen und auf die Parameter einwirken. Nur das konsequente Verfolgen dieser Unsicherheit wird es erlauben unter Berücksichtigung aller zur Modellierung notwendigen statistischen Momente das adäquate Distanzmass im Parameterraum zu definieren und den jetzt noch heuristisch gefundenen Schwellwert $\varepsilon$ herzuleiten. Im Interesse grösstmöglicher Präzision kann ausserdem unter Einbezug der Intensität des reflektierten Lichtstrahls, welches ein Mass für die Sicherheit eines jeden Messpunktes ist, ein gewichtendes leastsquare-fit-Verfahren ins Auge gefasst werden. Unentbehrliche Grundlage hierfür ist ein statistisches Modell, welches das Sensorrauschen genügend gut charakterisiert.

In Vorbereitung befindet sich ein darauf aufbauendes Map Building. Die Exploration soll unter Ausnutzung der symbolischen Strings arbeiten. Die geometrische Beschreibung eröffnet zudem die Möglichkeit der autonomen Konstruktion eines Graphen.

Die Erkennung von Türen und Behandlung von "cluttered areas" ist Voraussetzung für ein allgemeines Map Building und muss angegangen werden. Dies wurde bis jetzt ausgeklammert, weil dabei zwangsläufig auf der Ebene der Rohdaten gearbeitet werden muss und robotergeometrieabhängige Gesichtspunkte ins Spiel kommen. In diesem Sinne bildet das hier vorgestellte Verfahren zur Situationsbeschreibung eine geschlossene Einheit.

\section{Literaturverzeichnis}

[ARRAS96] Arras K.O., “Map Building”, Diplomarbeit am Institut für Robotik, ETH Zürich, 1996.

[HARTIG75] Hartigan J.A., “Clustering Algorithms”, Wiley, New York, 1975.

[KAISER95] Kaiser O., Pfiffner R., Vestli S.J., Astolfi A., "Positionsregelung für nichtholonome mobile Roboter”, 11. Fachgespräche Autonome Mobile Systeme, Karlsruhe, Deutschland, Nov. 1995.

[KanA89] Kanayama Y., "Spatial Learning by an Autonomous Mobile Robot with Ultrasonic Sensors", Technical Report of the University of California at Santa Barbara, Departement of Computer Science, TRCS89-06, February 1989.

[KuIPERS88] Kuipers B.J., Byun Y.T., "A Robust, Qualitative Approach to a Spatial Learning Mobile Robot", Proc. of the SPIE, Sensor Fusion: Spatial Reasoning and Scene Interpretation, Vol. 1003, 1988.

[LEON92] Leonard J.J., Durrant-Whyte H.F., "Directed Sonar Sensing for Mobile Robot Navigation", Kluwer Academic Publishers, 1992.

[SPÄTH83] Späth H., "Cluster-Formation und-Analyse", R. Oldenburg Verlag, 1983.

[VESTLI95] Vestli S.J., "Fast, accurate and robust estimation of mobile robot position and orientation", Doctoral Thesis Nr. 11360, ETH Zürich, 1995. 